\title{
Trade-off Geometries and Frequency-dependent Selection
}

Claire de Mazancourt (c.mazancourt@imperial.ac.uk)

Ulf Dieckmann (dieckmann @iiasa.ac.at)

\section{Approved by}

Leen Hordijk

Director, IIASA

August 2004

Interim Reports on work of the International Institute for Applied Systems Analysis receive only limited review. Views or opinions expressed herein do not necessarily represent those of the Institute, its National Member Organizations, or other organizations supporting the work. 


\section{IIASA STUDIES IN ADAPTIVE DYNAMICS No. 87}

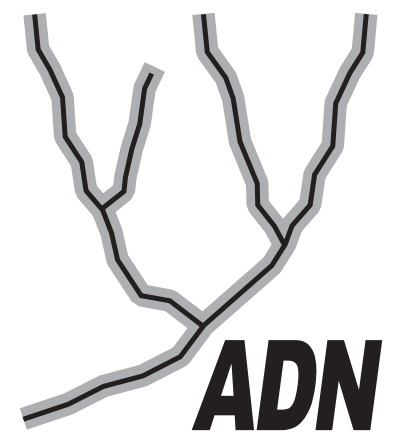

The Adaptive Dynamics Network at IIASA fosters the development of new mathematical and conceptual techniques for understanding the evolution of complex adaptive systems.

Focusing on these long-term implications of adaptive processes in systems of limited growth, the Adaptive Dynamics Network brings together scientists and institutions from around the world with IIASA acting as the central node.

Scientific progress within the network is collected in the IIASA Studies in Adaptive Dynamics series.

No. 1 Metz JAJ, Geritz SAH, Meszéna G, Jacobs FJA, van Heerwaarden JS: Adaptive Dynamics: A Geometrical Study of the Consequences of Nearly Faithful Reproduction. IIASA Working Paper WP-95-099 (1995). van Strien SJ, Verduyn Lunel SM (eds): Stochastic and Spatial Structures of Dynamical Systems, Proceedings of the Royal Dutch Academy of Science (KNAW Verhandelingen), North Holland, Amsterdam, pp. 183-231 (1996).

No. 2 Dieckmann U, Law R: The Dynamical Theory of Coevolution: A Derivation from Stochastic Ecological Processes. IIASA Working Paper WP-96-001 (1996). Journal of Mathematical Biology 34:579-612 (1996).

No. 3 Dieckmann U, Marrow P, Law R: Evolutionary Cycling of Predator-PreyInteractions: Population Dynamics and the Red Queen. IIASA Preprint (1995). Journal of Theoretical Biology 176:91-102 (1995).

No. 4 Marrow P, Dieckmann U, Law R: Evolutionary Dynamics of Predator-Prey Systems: An Ecological Perspective. IIASA Working Paper WP-96-002 (1996). Journal of Mathematical Biology 34:556-578 (1996).

No. 5 Law R, Marrow P, Dieckmann U: On Evolution under Asymmetric Competition. IIASA Working Paper WP-96-003 (1996). Evolutionary Ecology 11:485-501 (1997).

No. 6 Metz JAJ, Mylius SD, Diekmann O: When Does Evolution Optimize? On the Relation Between Types of Density Dependence and Evolutionarily Stable Life History Parameters. IIASA Working Paper WP-96-004 (1996).

No. 7 Ferrière R, Gatto M: Lyapunov Exponents and the Mathematics of Invasion in Oscillatory or Chaotic Populations. Theoretical Population Biology 48:126-171 (1995).

No. 8 Ferrière R, Fox GA: Chaos and Evolution. IIASA Preprint (1996). Trends in Ecology and Evolution 10:480485 (1995)

No. 9 Ferrière R, Michod RE: The Evolution of Cooperation in Spatially Heterogeneous Populations. IIASA Working Paper WP-96-029 (1996). The American Naturalist 147:692717 (1996).

No. 10 van Dooren TJM, Metz JAJ: Delayed Maturation in Temporally Structured Populations with Non-Equilibrium Dynamics. IIASA Working Paper WP-96-070 (1996). Journal of Evolutionary Biology 11:41-62 (1998).
No. 11 Geritz SAH, Metz JAJ, Kisdi É, Meszéna G: The Dynamics of Adaptation and Evolutionary Branching. IIASA Working Paper WP-96-077 (1996). Physical Review Letters 78:2024-2027 (1997).

No. 12 Geritz SAH, Kisdi É, Meszéna G, Metz JAJ: Evolutionary Singular Strategies and the Adaptive Growth and Branching of the Evolutionary Tree. IIASA Working Paper WP-96-114 (1996). Evolutionary Ecology 12:35-57 (1998).

No. 13 Heino M, Metz JAJ, Kaitala V: Evolution of Mixed Maturation Strategies in Semelparous Life-Histories: The Crucial Role of Dimensionality of Feedback Environment. IIASA Working Paper WP-96-126 (1996). Philosophical Transactions of the Royal Society of London Series B 352:1647-1655 (1997).

No. 14 Dieckmann U: Can Adaptive Dynamics Invade? IIASA Working Paper WP-96-152 (1996). Trends in Ecology and Evolution 12:128-131 (1997).

No. 15 Meszéna G, Czibula I, Geritz SAH: Adaptive Dynamics in a 2-Patch Environment: A Simple Model for Allopatric and Parapatric Speciation. IIASA Interim Report IR-97-001 (1997). Journal of Biological Systems 5:265-284 (1997).

No. 16 Heino M, Metz JAJ, Kaitala V: The Enigma of Frequency-Dependent Selection. IIASA Interim Report IR97-061 (1997). Trends in Ecology and Evolution 13:367-370 (1998).

No. 17 Heino M: Management of Evolving Fish Stocks. IIASA Interim Report IR-97-062 (1997). Canadian Journal of Fisheries and Aquatic Sciences 55:1971-1982 (1998).

No. 18 Heino M: Evolution of Mixed Reproductive Strategies in Simple Life-History Models. IIASA Interim Report IR-97063 (1997).

No. 19 Geritz SAH, van der Meijden E, Metz JAJ: Evolutionary Dynamics of Seed Size and Seedling Competitive Ability. IIASA Interim Report IR-97-071 (1997). Theoretical Population Biology 55:324-343 (1999).

No. 20 Galis F, Metz JAJ: Why Are There So Many Cichlid Species? On the Interplay of Speciation and Adaptive Radiation. IIASA Interim Report IR-97-072 (1997). Trends in Ecology and Evolution 13:1-2 (1998). 
No. 21 Boerlijst MC, Nowak MA, Sigmund K: Equal Pay for all Prisoners/ The Logic of Contrition. IIASA Interim Report IR-97-073 (1997). American Mathematical Society Monthly 104:303-307 (1997). Journal of Theoretical Biology 185:281-293 (1997).

No. 22 Law R, Dieckmann U: Symbiosis Without Mutualism and the Merger of Lineages in Evolution. IIASA Interim Report IR-97-074 (1997). Proceedings of the Royal Society of London Series B 265:1245-1253 (1998).

No. 23 Klinkhamer PGL, de Jong TJ, Metz JAJ: Sex and Size in Cosexual Plants. IIASA Interim Report IR-97-078 (1997). Trends in Ecology and Evolution 12:260-265 (1997).

No. 24 Fontana W, Schuster P: Shaping Space: The Possible and the Attainable in RNA Genotype-Phenotype Mapping. IIASA Interim Report IR-98-004 (1998). Journal of Theoretical Biology 194:491-515 (1998).

No. 25 Kisdi É, Geritz SAH: Adaptive Dynamics in Allele Space: Evolution of Genetic Polymorphism by Small Mutations in a Heterogeneous Environment. IIASA Interim Report IR-98-038 (1998). Evolution 53:993-1008 (1999).

No. 26 Fontana W, Schuster P: Continuity in Evolution: On the Nature of Transitions. IIASA Interim Report IR-98-039 (1998). Science 280:1451-1455 (1998).

No. 27 Nowak MA, Sigmund K: Evolution of Indirect Reciprocity by Image Scoring/ The Dynamics of Indirect Reciprocity. IIASA Interim Report IR-98-040 (1998). Nature 393:573-577 (1998). Journal of Theoretical Biology 194:561574 (1998).

No. 28 Kisdi É: Evolutionary Branching Under Asymmetric Competition. IIASA Interim Report IR-98-045 (1998). Journal of Theoretical Biology 197:149-162 (1999).

No. 29 Berger U: Best Response Adaptation for Role Games. IIASA Interim Report IR-98-086 (1998).

No. 30 van Dooren TJM: The Evolutionary Ecology of Dominance-Recessivity. IIASA Interim Report IR-98-096 (1998). Journal of Theoretical Biology 198:519-532 (1999).

No. 31 Dieckmann U, O'Hara B, Weisser W: The Evolutionary Ecology of Dispersal. IIASA Interim Report IR-98-108 (1998). Trends in Ecology and Evolution 14:88-90 (1999).

No. 32 Sigmund K: Complex Adaptive Systems and the Evolution of Reciprocation. IIASA Interim Report IR-98-100 (1998). Ecosystems 1:444-448 (1998).

No. 33 Posch M, Pichler A, Sigmund K: The Efficiency of Adapting Aspiration Levels. IIASA Interim Report IR-98103 (1998). Proceedings of the Royal Society London Series B 266:1427-1435 (1999).

No. 34 Mathias A, Kisdi É: Evolutionary Branching and Coexistence of Germination Strategies. IIASA Interim Report IR-99-014 (1999).

No. 35 Dieckmann U, Doebeli M: On the Origin of Species by Sympatric Speciation. IIASA Interim Report IR-99-013 (1999). Nature 400:354-357 (1999).

No. 36 Metz JAJ, Gyllenberg M: How Should We Define Fitness in Structured Metapopulation Models? Including an Application to the Calculation of Evolutionarily Stable Dispersal Strategies. IIASA Interim Report IR-99-019 (1999). Proceedings of the Royal Society of London Series B 268:499508 (2001)
No. 37 Gyllenberg M, Metz JAJ: On Fitness in Structured Metapopulations. IIASA Interim Report IR-99-037 (1999). Journal of Mathematical Biology 43:545-560 (2001).

No. 38 Meszéna G, Metz JAJ: Species Diversity and Population Regulation: The Importance of Environmental Feedback Dimensionality. IIASA Interim Report IR-99-045 (1999).

No. 39 Kisdi É, Geritz SAH: Evolutionary Branching and Sympatric Speciation in Diploid Populations. IIASA Interim Report IR-99-048 (1999).

No. 40 Ylikarjula J, Heino M, Dieckmann U: Ecology and Adaptation of Stunted Growth in Fish. IIASA Interim Report IR-99-050 (1999). Evolutionary Ecology 13:433-453 (1999).

No. 41 Nowak MA, Sigmund K: Games on Grids. IIASA Interim Report IR-99-038 (1999). Dieckmann U, Law R, Metz JAJ (eds): The Geometry of Ecological Interactions: Simplifying Spatial Complexity, Cambridge University Press, Cambridge, UK, pp. 135-150 (2000).

No. 42 Ferrière R, Michod RE: Wave Patterns in Spatial Games and the Evolution of Cooperation. IIASA Interim Report IR-99-041 (1999). Dieckmann U, Law R, Metz JAJ (eds): The Geometry of Ecological Interactions: Simplifying Spatial Complexity, Cambridge University Press, Cambridge, UK, pp. 318-332 (2000).

No. 43 Kisdi É, Jacobs FJA, Geritz SAH: Red Queen Evolution by Cycles of Evolutionary Branching and Extinction. IIASA Interim Report IR-00-030 (2000). Selection 2:161176 (2001).

No. 44 Meszéna G, Kisdi É, Dieckmann U, Geritz SAH, Metz JAJ: Evolutionary Optimisation Models and Matrix Games in the Unified Perspective of Adaptive Dynamics. IIASA Interim Report IR-00-039 (2000). Selection 2:193-210 (2001).

No. 45 Parvinen K, Dieckmann U, Gyllenberg M, Metz JAJ: Evolution of Dispersal in Metapopulations with Local Density Dependence and Demographic Stochasticity. IIASA Interim Report IR-00-035 (2000). Journal of Evolutionary Biology $16: 143-153$ (2003).

No. 46 Doebeli M, Dieckmann U: Evolutionary Branching and Sympatric Speciation Caused by Different Types of Ecological Interactions. IIASA Interim Report IR-00-040 (2000). The American Naturalist 156:S77-S101 (2000).

No. 47 Heino M, Hanski I: Evolution of Migration Rate in a Spatially Realistic Metapopulation Model. IIASA Interim Report IR-00-044 (2000). The American Naturalist 157:495511 (2001).

No. 48 Gyllenberg M, Parvinen K, Dieckmann U: Evolutionary Suicide and Evolution of Dispersal in Structured Metapopulations. IIASA Interim Report IR-00-056 (2000). Journal of Mathematical Biology 45:79-105 (2002).

No. 49 van Dooren TJM: The Evolutionary Dynamics of Direct Phenotypic Overdominance: Emergence Possible, Loss Probable. IIASA Interim Report IR-00-048 (2000). Evolution 54: 1899-1914 (2000).

No. 50 Nowak MA, Page KM, Sigmund K: Fairness Versus Reason in the Ultimatum Game. IIASA Interim Report IR00-57 (2000). Science 289:1773-1775 (2000).

No. 51 de Feo O, Ferrière R: Bifurcation Analysis of Population Invasion: On-Off Intermittency and Basin Riddling. IIASA Interim Report IR-00-074 (2000). International Journal of Bifurcation and Chaos 10:443-452 (2000). 
No. 52 Heino M, Laaka-Lindberg S: Clonal Dynamics and Evolution of Dormancy in the Leafy Hepatic Lophozia Silvicola. IIASA Interim Report IR-01-018 (2001). Oikos 94:525-532 (2001).

No. 53 Sigmund K, Hauert C, Nowak MA: Reward and Punishment in Minigames. IIASA Interim Report IR-01-031 (2001). Proceedings of the National Academy of Sciences of the USA 98:10757-10762(2001).

No. 54 Hauert C, De Monte S, Sigmund K, Hofbauer J: Oscillations in Optional Public Good Games. IIASA Interim Report IR-01-036 (2001).

No. 55 Ferrière R, Le Galliard J: Invasion Fitness and Adaptive Dynamics in Spatial Population Models. IIASA Interim Report IR-01-043 (2001). Clobert J, Dhondt A, Danchin E, Nichols J (eds): Dispersal, Oxford University Press, pp. 57-79 (2001).

No. 56 de Mazancourt C, Loreau M, Dieckmann U: Can the Evolution of Plant Defense Lead to Plant-Herbivore Mutualism. IIASA Interim Report IR-01-053 (2001). The American Naturalist 158: 109-123 (2001).

No. 57 Claessen D, Dieckmann U: Ontogenetic Niche Shifts and Evolutionary Branching in Size-Structured Populations. IIASA Interim Report IR-01-056 (2001). Evolutionary Ecology Research 4:189-217 (2002).

No. 58 Brandt H: Correlation Analysis of Fitness Landscapes. IIASA Interim Report IR-01-058 (2001).

No. 59 Dieckmann U: Adaptive Dynamics of Pathogen-Host Interacations. IIASA Interim Report IR-02-007 (2002). Dieckmann U, Metz JAJ, Sabelis MW, Sigmund K (eds): Adaptive Dynamics of Infectious Diseases: In Pursuit of Virulence Management, Cambridge University Press, Cambridge, UK, pp. 39-59 (2002).

No. 60 Nowak MA, Sigmund K: Super- and Coinfection: The Two Extremes. IIASA Interim Report IR-02-008 (2002). Dieckmann U, Metz JAJ, Sabelis MW, Sigmund K (eds): Adaptive Dynamics of Infectious Diseases: In Pursuit of Virulence Management, Cambridge University Press, Cambridge, UK, pp. 124-137 (2002).

No. 61 Sabelis MW, Metz JAJ: Perspectives for Virulence Management: Relating Theory to Experiment. IIASA Interim Report IR-02-009 (2002). Dieckmann U, Metz JAJ, Sabelis MW, Sigmund K (eds): Adaptive Dynamics of Infectious Diseases: In Pursuit of Virulence Management, Cambridge University Press, Cambridge, UK, pp. 379-398 (2002).

No. 62 Cheptou P, Dieckmann U: The Evolution of SelfFertilization in Density-Regulated Populations . IIASA Interim Report IR-02-024 (2002). Proceedings of the Royal Society of London Series B 269:1177-1186 (2002).

No. 63 Bürger R: Additive Genetic Variation Under Intraspecific Competition and Stabilizing Selection: A Two-Locus Study. IIASA Interim Report IR-02-013 (2002). Theoretical Population Biology 61:197-213 (2002).

No. 64 Hauert C, De Monte S, Hofbauer J, Sigmund K: Volunteering as Red Queen Mechanism for Co-operation in Public Goods Games. IIASA Interim Report IR-02-041 (2002). Science 296:1129-1132(2002).

No. 65 Dercole F, Ferrière R, Rinaldi S: Ecological Bistability and Evolutionary Reversals under Asymmetrical Competition. IIASA Interim Report IR-02-053 (2002). Evolution 56:1081-1090 (2002).
No. 66 Dercole F, Rinaldi S: Evolution of Cannibalistic Traits: Scenarios Derived from Adaptive Dynamics. IIASA Interim Report IR-02-054 (2002). Theoretical Population Biology 62:365-374 (2002).

No. 67 Bürger R, Gimelfarb A: Fluctuating Environments and the Role of Mutation in Maintaining Quantitative Genetic Variation. IIASA Interim Report IR-02-058 (2002). Genetical Research 80:31-46 (2002).

No. 68 Bürger R: On a Genetic Model of Intraspecific Competition and Stabilizing Selection. IIASA Interim Report IR02-062 (2002). Amer. Natur. 160:661-682 (2002).

No. 69 Doebeli M, Dieckmann U: Speciation Along Environmental Gradients. IIASA Interim Report IR-02-079 (2002). Nature 421:259-264 (2003).

No. 70 Dercole F, Irisson J, Rinaldi S: Bifurcation Analysis of a Prey-Predator Coevolution Model. IIASA Interim Report IR-02-078 (2002). SIAM Journal on Applied Mathematics 63:1378-1391 (2003).

No. 71 Le Galliard J, Ferrière R, Dieckmann U: The Adaptive Dynamics of Altruism in Spatially Heterogeneous Populations. IIASA Interim Report IR-03-006 (2003). Evolution 57:1-17 (2003).

No. 72 Taborsky B, Dieckmann U, Heino M: Unexpected Discontinuities in Life-History Evolution under SizeDependent Mortality. IIASA Interim Report IR-03-004 (2003). Proceedings of the Royal Society of London Series B 270:713-721 (2003).

No. 73 Gardmark A, Dieckmann U, Lundberg P: LifeHistory Evolution in Harvested Populations: The Role of Natural Predation. IIASA Interim Report IR-03-008 (2003). Evolutionary Ecology Research 5:239-257 (2003).

No. 74 Mizera F, Meszéna G: Spatial Niche Packing, Character Displacement and Adaptive Speciation Along an Environmental Gradient. IIASA Interim Report IR-03-062 (2003). Evolutionary Ecology Research 5: 363-382 (2003).

No. 75 Dercole F: Remarks on Branching-Extinction Evolutionary Cycles. IIASA Interim Report IR-03-075 (2003). Journal of Mathematical Biology 47: 569-580 (2003).

No. 76 Hofbauer J, Sigmund K: Evolutionary Game Dynamics. IIASA Interim Report IR-03-078 (2003). Bulletin of the American Mathematical Society 40: 479-519 (2003).

No. 77 Ernande B, Dieckmann U, Heino M: Adaptive Changes in Harvested Populations: Plasticity and Evolution of Age and Size at Maturation. IIASA Interim Report IR-03058 (2003).

No. 78 Hanski I, Heino M: Metapopulation-Level Adaptation of Insect Host Plant Preference and Extinction-Colonization Dynamics in Heterogeneous Landscapes. IIASA Interim Report IR-03-028 (2003). Theoretical Population Biology 63:309-338 (2003).

No. 79 van Doorn G, Dieckmann U, Weissing FJ: Sympatric Speciation by Sexual Selection: A Critical Re-Evaluation. IIASA Interim Report IR-04-003 (2004).

No. 80 Egas M, Dieckmann U, Sabelis MW: Evolution Restricts the Coexistence of Specialists and Generalists - the Role of Trade-off Structure. IIASA Interim Report IR-04-004 (2004). 
No. 81 Ernande B, Dieckmann U: The Evolution of Phenotypic Plasticity in Spatially Structured Environments: Implications of Intraspecific Competition, Plasticity Costs, and Environmental Characteristics. IIASA Interim Report IR-04-006 (2004). Journal of Evolutionary Biology (2004).

No. 82 Cressman R, Hofbauer J: Measure Dynamics on a One-Dimensional Continuous Trait Space: Theoretical Foundations for Adaptive Dynamics. IIASA Interim Report IR04-016 (2004).

No. 83 Cressman R: Dynamic Stability of the Replicator Equation with Continuous Strategy Space. IIASA Interim Report IR-04-017 (2004).

No. 84 Ravigné V, Olivieri I, Dieckmann U: Implications of
Habitat Choice for Protected Polymorphisms. IIASA Interim Report IR-04-005 (2004). Evolutionary Ecology Research 6: 125-145 (2004).

No. 85 Nowak MA, Sigmund K: Evolutionary Dynamics of Biological Games. IIASA Interim Report IR-04-013 (2004). Science 303: 793-799 (2004).

No. 86 Vukics A, Asbóth J, Meszéna G: Speciation in Multidimensional Evolutionary Space. IIASA Interim Report IR-04-028 (2004). Physical Review E 684 (2004).

No. 87 de Mazancourt C, Dieckmann U: Trade-off Geometries and Frequency-dependent Selection. IIASA Interim Report IR-04-039 (2004).

Issues of the IIASA Studies in Adaptive Dynamics series can be obtained at www.iiasa.ac.at/Research/ADN/Series.html or by writing to adn@iiasa.ac.at. 


\section{Contents}

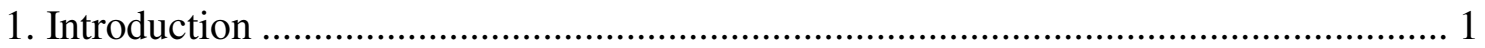

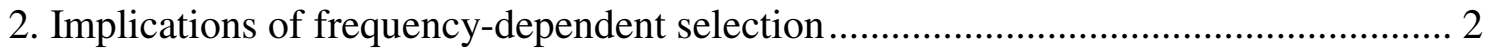

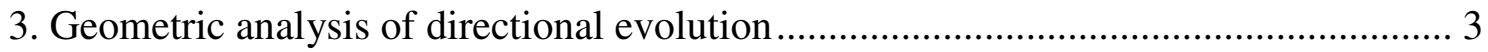

4. Geometric analysis of convergence stability and evolutionary stability ..................... 6

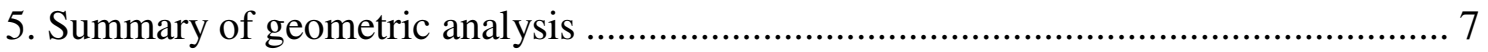

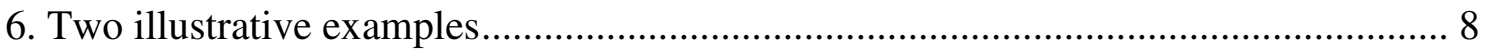

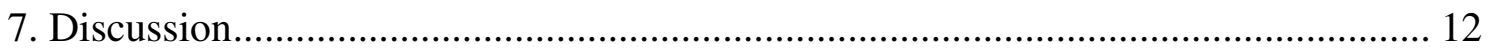

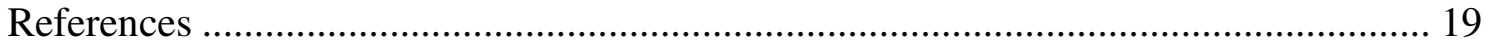

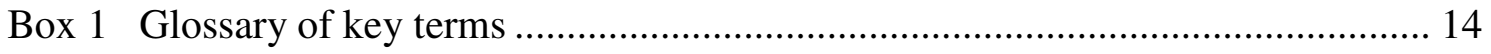

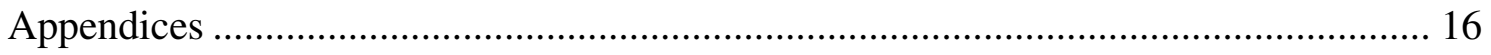

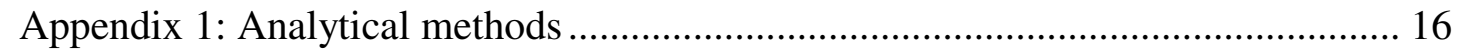

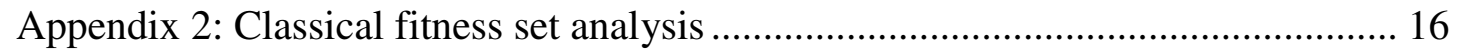

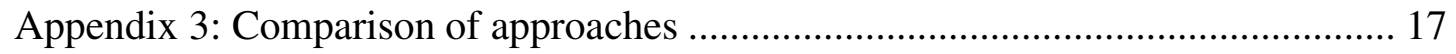




\begin{abstract}
Life-history evolution is determined by the interplay between natural selection and adaptive constraints. The classical approach to studying constrained life-history evolution - Richard Levins's geometric comparison of fitness sets and adaptive functions - is applicable when selection pressures are frequency-independent. Here we extend this widely used tool to frequency-dependent selection. Such selection pressures vary with a population's phenotypic composition, and are increasingly recognized as ubiquitous. Under frequency dependence, two independent properties have to be distinguished: evolutionary stability (an evolutionary stable strategy cannot be invaded once established) and convergence stability (only a convergence stable strategy can be attained through small, selectively advantageous steps). Combination of both properties results in four classes of possible evolutionary outcomes. We introduce a geometric mode of analysis that enables us to predict, for any bivariate selection problem, (i) evolutionary outcomes induced by trade-offs of given shape, (ii) shapes of trade-offs required for given evolutionary outcomes, (iii) the set of all evolutionary outcomes trade-offs can induce, (iv) effects of ecological parameters on evolutionary outcomes independent of trade-off shape.
\end{abstract}




\title{
About the Authors
}

\author{
Claire de Mazancourt \\ Department of Biological Sciences and \\ NERC Centre for Population Biology \\ Imperial College at Silwood Park \\ Ascot, Berkshire \\ SL 5 7PY, United Kingdom
}

Ulf Dieckmann

Adaptive Dynamics Network

International Institute for Applied Systems Analysis

Schlossplatz 1

A-2361 Laxenburg, Austria

\section{Acknowledgment}

Ulf Dieckmann gratefully acknowledges financial support by the Austrian Science Fund; by the Austrian Federal Ministry of Education, Science, and Cultural Affairs; and by the European Research Training Network ModLife (Modern Life-History Theory and its Application to the Management of Natural Resources), funded through the Human Potential Programme of the European Commission. We thank Ben Clark, Per Lundberg, David Murrell, and two anonymous reviewers for helpful comments on a previous version of this manuscript. 


\title{
Trade-off Geometries and Frequency-dependent Selection
}

\author{
Claire de Mazancourt \\ Ulf Dieckmann
}

\section{Introduction}

Evolution in general is driven by a complex interplay between selection pressures and constraints. To illustrate the importance of life-history constraints, Richard Law (1979) introduced the "Darwinian demon": exempt from all constraints, it produces very large numbers of offspring at frequent intervals, supplies each offspring with massive food reserves, lives long, achieves this in any habitat, etc. Evidently, such imaginary superorganism would quickly take over the earth's biosphere and eradicate all diversity. Understanding biodiversity therefore always entails identifying the trade-offs and constraints that prevent Darwinian demons from existing in nature.

Some constraints arise from mathematical or physical considerations; for example, a probability is bounded between 0 and 1. Other constraints such as trade-offs are often unknown, and even when qualitative insight exists, their shapes remain uncertain: empirical measurements of plausible trade-offs are amazingly difficult (e.g., Simms and Rausher 1987; Mole 1994; Ebert and Bull 2003). Yet, traditional evolutionary predictions tend to crucially depend on the precise shape of trade-offs assumed (e.g., Lipsitch 1996; Kisdi 2001; Dieckmann 2002). It is therefore critical to develop tools for evolutionary analysis that are robust against trade-off variations. Here we introduce a geometric mode of analysis that yields general conclusions independent of particular trade-off shapes.

The fundamental importance of natural selection's interaction with trade-offs has been highlighted by Richard Levins's fitness set analysis. Introduced already forty years ago (Levins 1962a, 1962b, 1968), this geometric method for predicting evolutionary outcomes in bivariate selection problems still enjoys widespread recognition - not only in evolutionary teaching (e.g., Yodzis 1989:324-351; Case 1999:175-177; Calow 1999:758) but also in research (Levins 1962a and 1968 have accrued more than 1000 citations over the past 15 years). Levins's geometric analysis is popular because: (i) it is simple and geometric, (ii) it separately considers selection pressures and trade-offs, thus allowing them to be varied independently and flexibly, and (iii) it allows addressing questions about coexistence and the emergence of polymorphisms.

Yet Levins's approach suffers from a serious drawback: it does not allow for selection pressures to be frequency-dependent. This is because, in Levins's framework, each combination of phenotypes is assigned a unique fitness value - independently of which other phenotypes reside in the adapting population. However, in general, the 
lifetime reproductive success of an individual does not only depend on its own phenotype, but also on the environment it experiences, notably including the conspecifics and heterospecifics it interacts with. For example, the effect of competitors on a focal individual depends on their number, but also on their phenotypes. Based on a great variety of such examples, it seems fair to conclude that most natural selection pressures possess frequency-dependent components. This paper extends classical fitness set analysis to include frequency-dependent selection.

Under frequency dependent selection, two independent properties have to be distinguished, referred to as evolutionary stability (an evolutionary stable strategy cannot be invaded once established) and convergence stability (only a convergence stable strategy can be attained through small, selectively advantageous steps). These two properties are independent (Eshel 1983), resulting in four classes of possible evolutionary outcomes (see glossary). Finding and classifying evolutionary outcomes along a given trade-off curve is a one-dimensional problem that is already thoroughly understood. However, the existing methods require making ad-hoc assumptions about the shape of trade-off curves (Rueffler et al. 2004). Our method, by contrast, provides a geometric representation of evolutionary stability and convergence stability. It thus enables us to visualize in a single go how evolutionary outcomes depend on trade-offs. This means that we can predict the evolutionary outcome induced by any specific tradeoff and delineate the features of trade-off curves required for inducing a specific evolutionary outcome. In addition, we can easily identify the complete set of evolutionary outcomes conceivable trade-offs can induce and ascertain the effect of ecological parameters on evolutionary outcomes independent of trade-off shapes. We illustrate the utility of such an approach by showing a situation where a whole class of possible outcomes had been overlooked until now because only particular trade-offs were investigated (see first example below). By cleanly separating the analysis of selection pressures from the analysis of trade-off effects, we thus obtain general results without any danger of accidentally overlooking evolutionary outcomes that become possible only for particular types of trade-off.

Our geometric approach is complemented by corresponding analytical methods (Appendix 1): to apply the toolbox presented here, readers can switch between these two equivalent styles of analysis. Section 2 introduces the feedback between individual fitness and population composition resulting in frequency-dependent selection. Sections 3 and 4 describe the core of our generalized framework and explain how the intricacies of frequency-dependent selection are accommodated in a simple geometrical style of analysis. The resulting technique is summarized in Section 5. Section 6 illustrates how to use the new graphical tools to analyze adaptations to two habitats. We also explain how Levins's classical fitness set analysis (Appendix 2) is encompassed by our new framework (Appendix 3). A glossary of key technical terms accompanies this article (Box 1).

\section{Implications of frequency-dependent selection}

Frequency-dependent selection is ubiquitous in nature, since the performance of organisms is rarely independent of the phenotypes prevalent in their population. We 
already mentioned competitive interactions, where the phenotypes against which a focal individual is competing will usually matter (e.g., Taper and Case 1985; Matsuda and Abrams 1994; Law et al. 1997; Kisdi 1999). Other key examples of frequencydependent selection involve cheating or defecting strategies in behavioral evolution. Such strategies may do very well as long as they are rare in a population of cooperative individuals but fare worse or even perish when surrounded only by other cheaters (e.g., Nowak et al. 1994; Hauert et al. 2002; Sigmund 2002; Le Galliard et al. 2003). Other mechanisms resulting in frequency-dependent selection are predator-prey interactions (e.g., Saloniemi 1993; Dieckmann et al. 1995; Abrams and Matsuda 1996), plantherbivore dynamics (e.g., Harding and Lovelock 1996; de Mazancourt et al. 2001), mutualism (e.g., Mallet 1999; Doebeli and Dieckmann 2000), and epidemiological processes (e.g., May 1983; Dieckmann 2002). Hence, for many problems of interest in life-history evolution, a unique fitness value cannot be attributed to each strategy independently of the composition of its population; consequently, Levins's framework of analysis is not applicable (see Appendix 2). It thus seems important to develop an extended framework for geometrically analyzing constrained life-history evolution under frequency dependence. In general, this leads to models in which the entire distribution of phenotypes in a population has to be tracked through evolutionary time (as, e.g., in Taper and Case 1992). Two simplifications are commonly used to tackle this complexity:

- Either one assumes that phenotypes in the evolving population always remain normally distributed and that they exhibit the same phenotypic variance at any moment in time (quantitative genetics approach; e.g., Lande 1979; Taper and Case 1985; Iwasa et al. 1991; Abrams et al. 1993; Vincent et al. 1993; Turelli and Barton 1994).

- Or one assumes that evolution proceeds by a sequence of evolutionary innovations during which a new selectively advantageous phenotype invades and replaces an essentially monomorphic population of resident phenotypes (adaptive dynamics approach; e.g., Metz et al. 1992, 1996; Dieckmann and Law 1996; Geritz et al. 1997, 1998; Dieckmann 1997).

In general, both approaches yield similar results. The former is more suited to study short-term evolution, where selection acts on substantial standing genetic variation generated through recombination, whereas the latter is more geared to analyzing longterm evolution, where evolution depends on new phenotypes becoming available through suitable mutations or rare recombination. This paper's Discussion highlights the one important situation in which evolutionary predictions could qualitatively vary with the choice of approach.

\section{Geometric analysis of directional evolution}

In phenotype space, a population is represented by a point whose location corresponds to the population's mean phenotype (in the quantitative genetic approach) or resident phenotype (in the adaptive dynamics approach). As the population's phenotypic composition changes over time through evolution and selection, such a point describes a trajectory in phenotype space. Without frequency dependence, a single fitness value can 
be ascribed to each phenotype, resulting in a fitness landscape (Lande 1976); a population then just climbs up its fitness landscape until it reaches a maximum. A single fitness value per phenotype does not exist under frequency dependence because fitness depends on the environment (and the environment, in turn, depends on the population's phenotypic composition). To analyze directional evolution under frequency dependence, the direction of selection has to be inferred from local selection gradients. These describe the direction most favored by selection around a population's mean phenotype (in the quantitative genetics approach) or resident phenotype (in the adaptive dynamics approach).

Calculating local selection gradients under frequency dependence relies on the key notion of invasion fitness. The invasion fitness $f\left(x^{\prime}, x\right)$ of a rare phenotype $x^{\prime}$ in the environment $E$ determined by a phenotype $x$ simply is the per capita growth rate of $x^{\prime}$ in $E$ (Metz et al. 1992; Rand et al. 1993; Ferrière and Gatto 1995). If $f\left(x^{\prime}, x\right)$ is positive, $x^{\prime}$ can invade into a population dominated by $x$, otherwise it cannot. In the quantitative genetics approach, $E$ is inferred from $x$ by assuming that a population's phenotypic distribution has mean $x$ and constant variance (e.g., Abrams et al. 1993). [Even though little used, more elaborate approaches do exist, in which also a population's variances and covariances are allowed to change dynamically, while other features of the phenotypic distribution are then kept constant instead (Taper and Case 1985; Iwasa et al. 1991; Vincent et al. 1993; Turelli and Barton 1994). The resulting models are relatively complex and therefore rarely applied.] The situation is simpler in the adaptive dynamics approach, where it is assumed that the resident population is essentially monomorphic at $x$, since, between evolutionary innovations, selection has sufficient time to weed out deleterious variation (e.g., Metz et al. 1996). The local selection gradient is defined as the direction around a resident phenotype towards which variant phenotypes have the highest invasion fitness. Once the invasion fitness of a specific model is known, its local selection gradient can be calculated as $g(x)=\partial f /\left.\partial x^{\prime}\right|_{x^{\prime}=x}$. For example, when considering two phenotypic components, $x=\left(x_{1}, x_{2}\right)$, the local selection gradient $g(x)=\left.\left(\partial f / \partial x_{1}^{\prime}, \partial f / \partial x_{2}^{\prime}\right)\right|_{x^{\prime}=x}$ is also a twodimensional vector, capturing the sensitivity of invasion fitness with regard to variations in the phenotypic components $x_{1}^{\prime}$ and $x_{2}^{\prime}$.

Average evolutionary change in the phenotype $x$ follows the local selection gradient, $d x / d t \infty g(x)$ [see Lande (1979) and Dieckmann and Law (1996) for derivations based, respectively, on quantitative genetics and adaptive dynamics; since we are, like Levins, primarily interested in geometric analyses of one-dimensional trade-off curves embedded in two-dimensional trait spaces, covariance constraints will usually not affect the evolutionary outcomes, as we explain in the Discussion]. By considering a tangent vector $h$ along a trade-off curve we can conclude that directional evolution ceases where $g \cdot h=0$, while evolution takes the phenotype in (against) the direction of $h$ as long as $g \cdot h>0(g \cdot h<0)$.

As a first geometric element of our extended toolbox we now introduce curves that, at any point in trait space, are orthogonal to the local selection gradient and hence to all resultant evolutionary trajectories. For any point on such a curve, the curve itself locally separates regions of phenotypes that can be reached though sequences of successful 


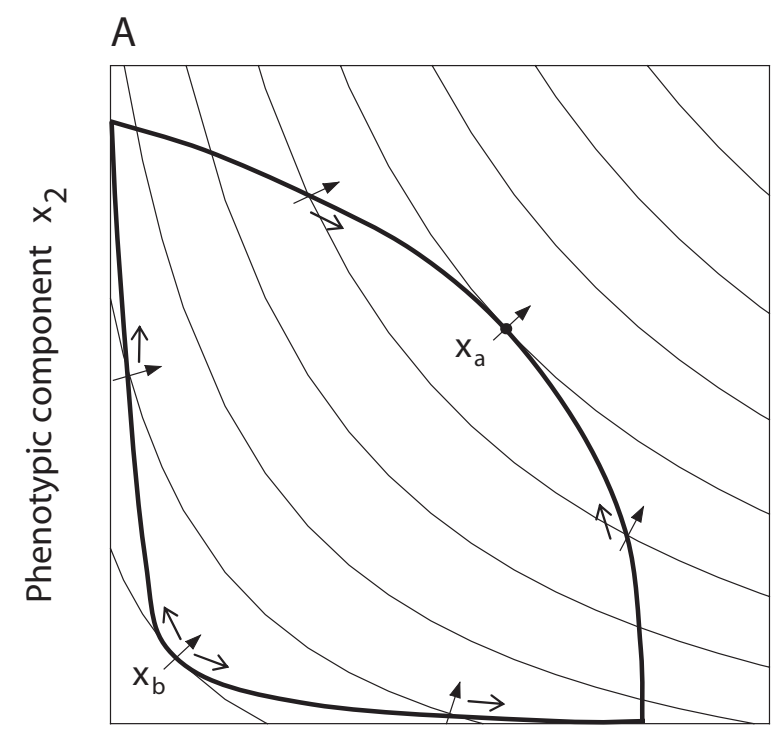

B

Phenotypic component $x_{1}$

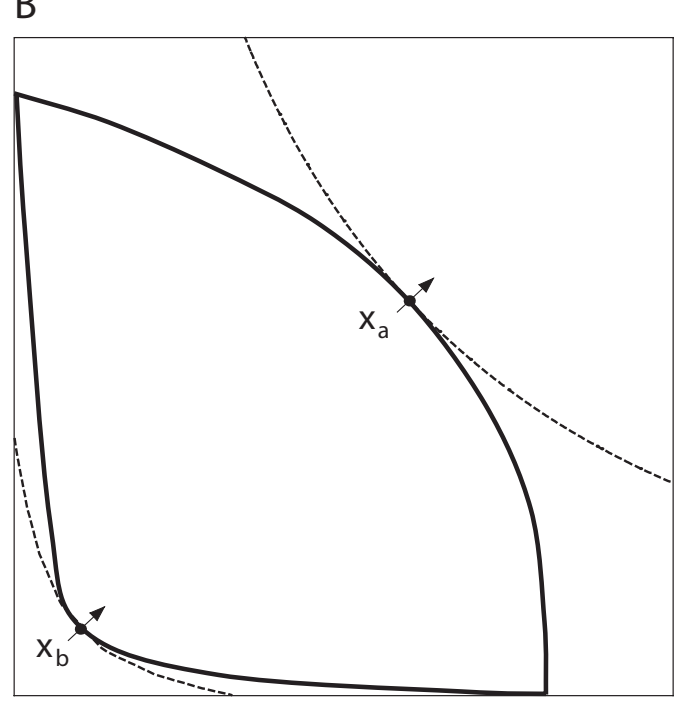

Figure 1 Geometric analysis of constrained frequency-dependent evolution. A-boundaries are shown as thin lines, I-boundaries as dashed lines, and trade-off curves as thick lines; arrows indicate the direction of the local selection gradient. (A) A-boundaries determine the direction of evolution along the trade-off curve. Along the two trade-off curves directional selection ceases at the evolutionarily singular strategies $x_{a}$ and $x_{b}$. The strategy $x_{a}$ is convergence stable (i.e., attainable through gradual evolution along the trade-off curve), whereas the strategy $x_{b}$ is not. (B) I-boundaries determine the evolutionary stability of a singular strategy. Two I-boundaries are drawn for the singular strategies $x_{a}$ and $x_{b}$ : these delimit the range of strategies that can invade the corresponding singular strategy. Accordingly, the strategy $x_{a}$ is locally evolutionarily stable (i.e., uninvasible by its neighbors along the trade-off curve), whereas the strategy $x_{b}$ is not. Section 5 contains a summary of how to use A-boundaries and I-boundaries.

invasions involving infinitesimal phenotypic steps, from phenotypes for which this is not possible. Accordingly, we refer to these curves as attainability boundaries, or Aboundaries for short.

A-boundaries allow us to determine geometrically which parts of trade-off curves are evolutionarily attracting, and how directional evolution proceeds once a trade-off curve has been reached, as illustrated in figure 1A. As far as directional evolution is concerned, A-boundaries thus assume exactly the role that contours of the adaptive function played in Levins's original approach; in the absence of frequency-dependent selection, these two types of curve simply coincide. By comparing A-boundaries and trade-off curves we can predict evolution's direction along the latter: the evolving phenotype slides along the trade-off curve until the two become parallel (figure 1A), or a boundary of the feasible trait space is reached. In other words, with $S_{A}$ and $S_{T}$ denoting, respectively, the slopes of A-boundaries and of trade-off curves, directional evolution ceases at points along a trade-off curve at which these two slopes coincide, $S_{A}=S_{T}$. Such points are called evolutionarily singular (Metz et al. 1996). 


\section{Geometric analysis of convergence stability and evolutionary stability}

When selection is frequency-dependent, evolutionarily singular points have two independent stability properties (Eshel and Motro 1981; Eshel 1983; Taylor 1989; Christiansen 1991; Takada and Kigami 1991). Local evolutionary stability implies that a phenotype cannot be invaded by any neighboring phenotypes, whereas convergence stability, or attainability, implies that directional evolution around a phenotype leads towards it. Consequently, four types of evolutionarily singular phenotype have to be distinguished (see glossary). (a) First are so-called continuously stable strategies (Eshel and Motro 1981; Eshel 1983), which are both locally evolutionarily stable and convergence stable, and thus serve as likely endpoints of gradual evolutionary change. (b) Second are invasible repellors, lacking both local evolutionary stability and convergence stability. (c) Third are so-called Garden-of-Eden configurations (Nowak and Sigmund 1989), which are locally evolutionarily stable (and hence uninvasible once attained) and at the same time evolutionarily repelling (and hence not attainable through small evolutionary steps). (d). Fourth are evolutionary branching points (Metz et al. 1992, 1996), which are convergence stable but lack local evolutionary stability. The latter configurations cause evolutionary convergence to disruptive selection, which can induce the phenotypic divergence of two subpopulations, resulting in a population-level dimorphism. In this paper we provide a geometric framework for analyzing how, under conditions of frequency-dependent selection, evolutionary outcomes of these four types depend on the shape and position of trade-off curves constraining the course of evolution. Notice that in models of frequency-independent evolution types (c) and (d) cannot occur.

The A-boundaries considered above allow for a simple geometric assessment of convergence stability. Singular phenotypes are evolutionary attractors (i.e., they are convergence stable or evolutionarily attainable) if their A-boundary shows that no neighboring phenotypes can be reached by directional evolution ( $x_{a}$ in figure 1A). Otherwise such phenotypes are evolutionary repellors (i.e., they are not convergence stable and thus evolutionarily unattainable; $x_{b}$ in figure $\left.1 \mathrm{~A}\right)$.

To assess evolutionary stability, we need to introduce a second type of curve: for any point in trait space, the invasibility boundary (I-boundary for short) separates regions of phenotypes that can invade into a population situated at that point from phenotypes that cannot. The I-boundary of a given point $x$ thus delineates its invader set, which includes all points $x^{\prime}$ with $f\left(x^{\prime}, x\right)>0$. Notice that the I- and A-boundaries of any point $x$ have identical slopes at $x, S_{I}=S_{A}$. A singular phenotype lying on a trade-off curve experiences disruptive selection if its invader set includes the surrounding trade-off curve ( $x_{b}$ in figure 1B). By contrast, if the trade-off curve does not fall into the invader set, selection on the singular phenotype is stabilizing $\left(x_{a}\right.$ in figure 1B).

Thus, the relative curvatures of A-boundaries, I-boundaries, and trade-off curves determine the mode of selection at evolutionarily singular phenotypes. Since, by definition, the local selection gradient at a singular phenotype is orthogonal to the local I-boundary, A-boundary, and trade-off curve, it is convenient to use the following 


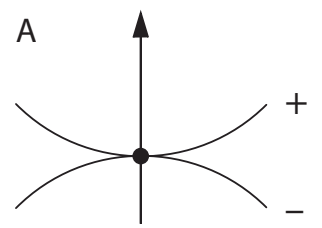

Sign convention for curvatures
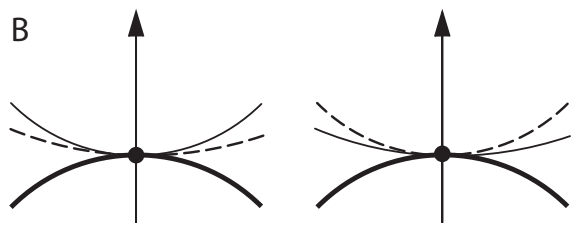

Continuously stable strategy: $\mathrm{C}_{\mathrm{T}}<\mathrm{C}_{\mathrm{A}}$ and $\mathrm{C}_{\mathrm{T}}<\mathrm{C}_{\mathrm{I}}$
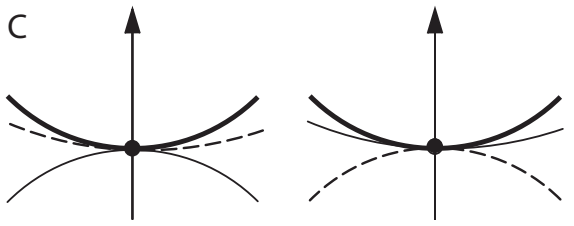

Invasible repellor:

$\mathrm{C}_{\mathrm{T}}>\mathrm{C}_{\mathrm{A}}$ and $\mathrm{C}_{\mathrm{T}}>\mathrm{C}_{\mathrm{I}}$

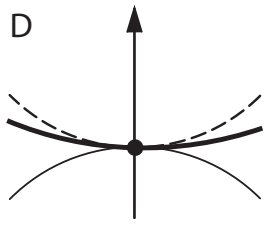

Garden -of-Eden: $\mathrm{C}_{\mathrm{A}}<\mathrm{C}_{\mathrm{T}}<\mathrm{C}_{\text {}}$

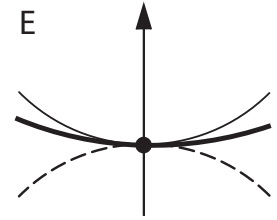

Evolutionary branching point: $C_{1}<C_{T}<C_{A}$

Figure 2 Geometric signatures of the four types of evolutionarily singular strategy possible under frequency-dependent selection along trade-off curves. A-boundaries are shown as thin lines, I-boundaries as dashed lines, and trade-off curves as thick lines; arrows indicate the direction of the local selection gradient. (A) Sign convention for curvatures, based on the direction of the local selection gradient. (B) Continuously stable strategy. (C) Invasible repellor. (D) Garden-of-Eden configuration. (E) Evolutionary branching point. Section 4 explains these four types in greater detail.

simple convention for defining the signs of their curvatures: when, looking along the local selection gradient, the curve is convex, it has positive curvature, and otherwise its curvature is negative. This convention is shown in figure $2 \mathrm{~A}$. Denoting curvatures by $C_{A}, C_{I}$, and $C_{T}$, respectively, we can thus conclude that a singular phenotype is convergence stable (unstable) if $C_{T}<C_{A}\left(C_{T}>C_{A}\right)$ and locally evolutionarily stable (unstable) if $C_{T}<C_{I}\left(C_{T}>C_{I}\right)$. In other words, a singular phenotype is evolutionarily stable (attainable) whenever the surrounding part of the trade-off curve does not fall in the region of trait space delineated by the I-boundary (A-boundary) into which the local selection gradient points. This result allows for a rapid visual assessment of evolutionary and convergence stability along one-dimensional trade-offs embedded in two-dimensional trait spaces. Analytical details corresponding to these geometric insights are provided in Appendix 1.

\section{Summary of geometric analysis}

With these geometric tools in place we can predict how outcomes of frequencydependent evolution depend on the shape of trade-off curves:

- Which parts of trade-off curves are attracting directional evolution is determined by a model's A-boundaries.

- Along a trade-off curve, directional evolution ceases where A-boundaries (and therefore also I-boundaries) have the same slope as the trade-off curve, $S_{A}=S_{I}=S_{T}$. 
- Depending on the curvatures of A-boundaries, I-boundaries, and the trade-off curve, such singular phenotypes possess evolutionary (convergence) stability if and only if $C_{T}<C_{I}\left(C_{T}<C_{A}\right)$.

Figure 2 illustrates the resultant geometric 'signatures' of the four types of evolutionarily singular phenotype, i.e., the relative ordering of the trade-off curve, Aboundary and I-boundary that leads to a specific evolutionary outcome. The case of singular phenotypes not lying on a trade-off curve but instead in the interior of the feasible trait space is briefly covered in Appendix 1; since selection is then unaffected by the focal trade-off, such cases are not further discussed here. Notice that Garden-ofEden configurations (figure 2D) and evolutionary branching points (figure 2E) require trade-off curves be sandwiched between the local A- and I-boundaries. This underscores that, if A- and I-boundaries coincide, as happens under frequency-independent selection, these types of evolutionarily singular strategy are ruled out.

It is particularly interesting to take a close look at the geometric conditions that can lead to population-level polymorphisms. To reach such polymorphisms gradually, evolution must converge on an evolutionary branching point, which requires $C_{I}<C_{T}<C_{A}$. We can therefore visualize the potential for evolutionary branching by depicting the difference $C_{A}-C_{I}$ through grayscales in trait space, with white indicating all negative values and zero, and with the darkest gray corresponding to the maximal difference and thus to the highest potential for evolutionary branching. This method is particularly useful for assessing through which parts of trait space a trade-off curve has to pass and which shape it has to possess there for it to induce a population-level polymorphism.

Notice that once the potential for dimorphism has been established, the corresponding dimorphic evolutionary attractor is determined by considering the invasion fitness $f\left(x^{\prime}, x_{a}, x_{b}\right)$ of phenotypes $x^{\prime}$ in a dimorphic population dominated by phenotypes $x_{a}$ and $x_{b}$. Analytical details are explained elsewhere (e.g., Geritz 1998) and no geometric method appears to exist as yet to replace the established analytical techniques.

\section{Two illustrative examples}

As a deliberately simple first example, we consider an annual organism in an environment consisting of two types of habitat, where the two probabilities $x_{i}$ to survive in habitat $i=1,2$ can change evolutionarily. While it is natural to assume that a trade-off exists between these survival probabilities, we do not wish to restrict ourselves to any ad-hoc choice. At the beginning of seasons, individuals are randomly distributed across habitats, and at the season's end a fixed number of randomly selected individuals are recruited from each habitat, with a fraction $c_{1}$ coming from habitat 1 and a fraction $c_{2}=1-c_{1}$ coming from habitat 2 . We now consider a phenotype $x^{\prime}$ at frequency $p$ in a population with resident phenotype $x$ at frequency $q=1-p$. At the end of the season, the frequency of $x^{\prime}$ is thus given by $\tilde{p}=c_{1} p x_{1}^{\prime} /\left(p x_{1}^{\prime}+q x_{1}\right)+c_{2} p x_{2}^{\prime} /\left(p x_{2}^{\prime}+q x_{2}\right)$. Phenotype $x^{\prime}$ increases in frequency if its per capita growth rate, $\log (\tilde{p} / p)$, is positive. The invasion fitness of $x^{\prime}$ in the environment set by $x$ is its per capita growth rate when rare $(p \approx 0), f\left(x^{\prime}, x\right)=\log \left(c_{1} x_{1}^{\prime} / x_{1}+c_{2} x_{2}^{\prime} / x_{2}\right)$. 

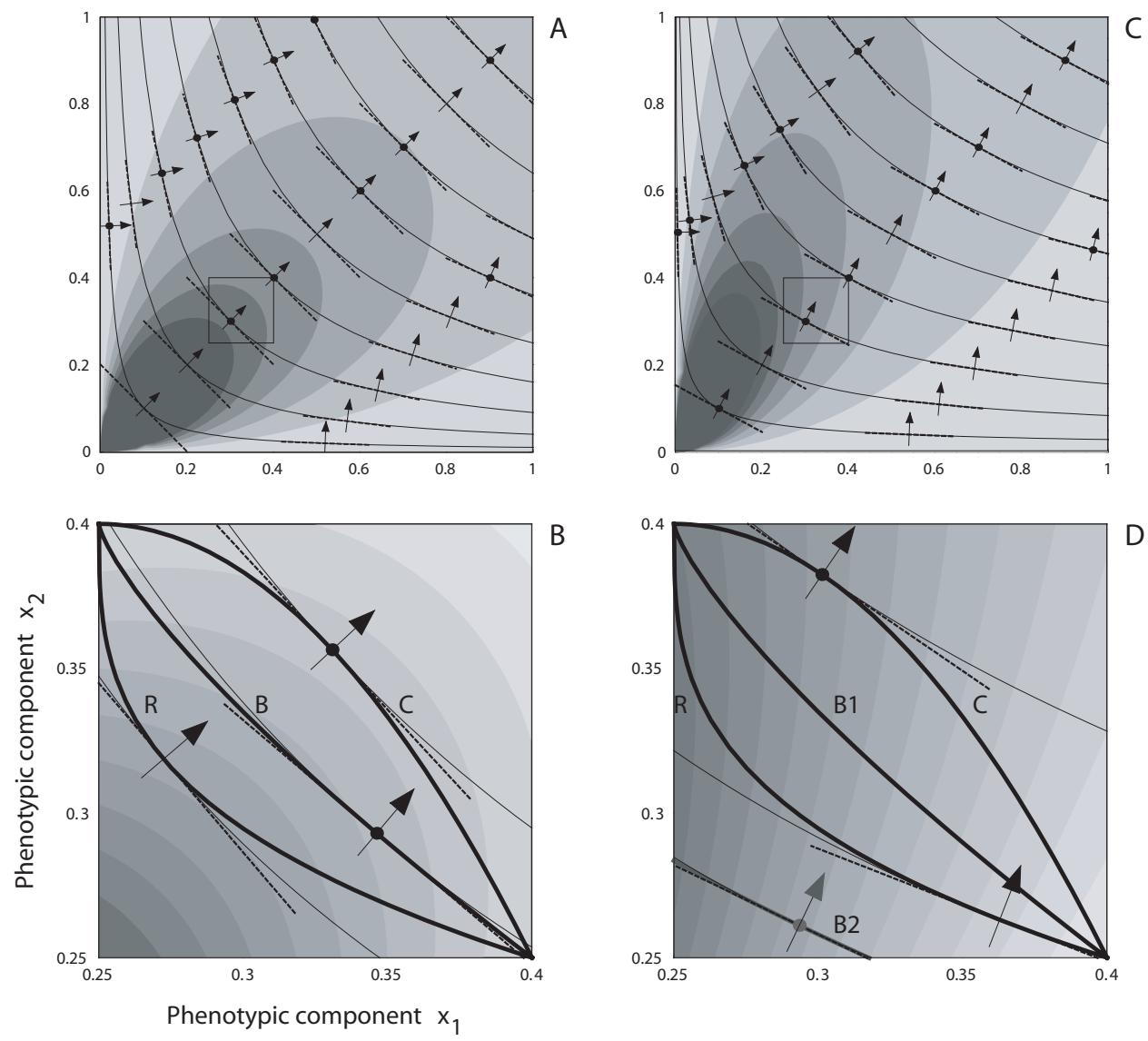

Figure 3 Geometric analysis of first example. The two phenotypic components $x_{1}$ and $x_{2}$ measure survival probabilities in two alternative habitats. A-boundaries are shown as thin lines, I-boundaries as dashed lines, and trade-off curves as thick lines; arrows indicate the direction of the local selection gradient. Shading visualizes the potential for evolutionary branching, as measured by $C_{A}-C_{I}$ (white: branching impossible, $C_{A}-C_{I} \leq 0$; darkest gray: highest potential for branching, $C_{A}-C_{I}>0$ ). (A) In the left column, the recruitment fraction from habitat 1 is $c_{1}=0.5$. Comparing the geometric signatures in this diagram to those in figure 2 shows that, in this model, all points $\left(x_{1}, x_{2}\right)$ can be alternatively turned into a continuously stable strategy, an invasible repellor, or an evolutionary branching point: this only requires the trade-off's shape be chosen appropriately. On the other hand, no trade-off whatsoever can induce a Garden-of-Eden configuration here. In addition, the potential for evolutionary branching turns out to be highest when the two survival probabilities $x_{1}$ and $x_{2}$ are low. (B) Enlargement of the boxed area in (A). For three different trade-off curves, the resulting evolutionarily singular strategies are shown by filled circles. Trade-off $\mathrm{R}$ induces an invasible repellor, trade-off $\mathrm{B}$ an evolutionary branching point, and trade-off $\mathrm{C}$ a continuously stable strategy. (C) In the right column, the recruitment fraction $c_{1}$ from habitat 1 has been reduced to $c_{1}=0.35$ : in response to this environmental change, A- and Iboundaries become shallower. The altered geometry implies that, for all trade-off curves, there now is a stronger selection pressure towards survival in habitat 2. Also, as shown by the shading, evolutionary branching now is more likely when survival in habitat 2 exceeds survival in habitat 1. (D) Enlargement of the boxed area in (C). Along trade-off $\mathrm{R}$, a larger set of initial phenotypes (left of the repellor) will now evolve toward high survival in habitat 2. Along trade-off B1, no evolutionary branching is possible any more; instead, evolution simply seeks to maximize survival in habitat 2. Trade-off B2 gives an example of a curve shape that induces evolutionary branching under the altered environmental conditions. The continuously stable strategy along trade-off curve $\mathrm{C}$ moves left and up, corresponding to enhanced survival in habitat 2 at the cost of diminished survival in habitat 1. Section 6 provides further details about the first example. 
Results of our geometric analysis are presented in figure 3. Inspection of diagrams like figures $3 \mathrm{~A}$ and $3 \mathrm{~B}$ reveals that for any value of $c_{1}$ any point in trait space can be turned into either a continuously stable strategy, an invasible repellor, or an evolutionary branching point, if only the trade-off between $x_{1}$ and $x_{2}$ is chosen appropriately. By contrast, in this model no choice of trade-off can ever bring about a Garden-of-Eden configuration. In addition, the distribution of grayscales highlights that evolutionary branching points become more likely if the evolutionarily singular survival probabilities induced by a trade-off are low and proportional to recruitment, $x_{1} / x_{2} \approx c_{1} / c_{2}$.

We can also easily draw the following comprehensive conclusions concerning the implications of particular trade-off shapes. A continuously stable strategy arises for all convex trade-offs (trade-offs $\mathrm{C}$ in figures $3 \mathrm{C}$ and 3D), and also for linear trade-offs, as used, e.g., by de Meeus and Goudet (2000). Slightly concave trade-offs will result in evolutionary branching points (trade-offs $\mathrm{B} 1$ in figure $3 \mathrm{C}$ and $\mathrm{B} 2$ in figure $3 \mathrm{D}$ ). It can even be shown (both analytically and geometrically) that the particular trade-off shape resulting from normally distributed utilization along a one-dimensional phenotypic axis, as used by Kisdi and Geritz (2001), can only lead to either continuously stable strategies or evolutionary branching points. By contrast, our geometric analysis shows that any trade-off that, at the singular phenotype it induces, is more concave than the local Aboundary brings about an invasible repellor (trade-offs $\mathrm{R}$ in figures $3 \mathrm{C}$ and $3 \mathrm{D}$ ). This last possibility was systematically overlooked in previous work on this model through which only particular trade-off families were analyzed.

An important additional feature of the geometric analysis is that it allows predicting, independently of trade-off shape, how environmental change affects evolutionary outcomes. The transition from figure $3 \mathrm{C}$ to figure $3 \mathrm{D}$ illustrates the effect of decreasing the fraction $c_{1}$ of individuals recruited from habitat 1: it results in shallower Aboundaries throughout trait space. Given the direction of the local selection gradient, this means that there will be selection for a higher survival in habitat 2 at the expense of a lower survival in habitat 1 , whatever the shape of the trade-off curve. Accordingly, along trade-off $\mathrm{C}$ the continuously stable strategy shifts left. Along the slightly concave trade-off B1, no slope matches the new A-boundaries such that the evolutionary branching point on B1 disappears and is replaced by directional evolution to the left (trade-off B2 illustrates that evolutionary branching points can yet be induced by a different trade-off shape). Finally, along the more strongly concave trade-off $R$, the invasible repellor shifts to the right, thus increasing the basin of attraction of the phenotype maximizing survival in habitat 2.

In our second example we consider an organism living in an environment consisting of two habitat types and possessing two phenotypic components $x_{i}, i=1,2$. This time the environment is aseasonal: individuals randomly encounter the two habitat types with probability $c_{i}$, experience the same intrinsic per capita growth rate $r$ in each habitat, and are density-regulated through symmetric Lotka-Volterra competition. The carrying capacity of phenotypes in each habitat differs according to $K_{i}(x)=k x_{i}$, where $k$ is a scaling factor. We assume that the competition coefficients between two different 

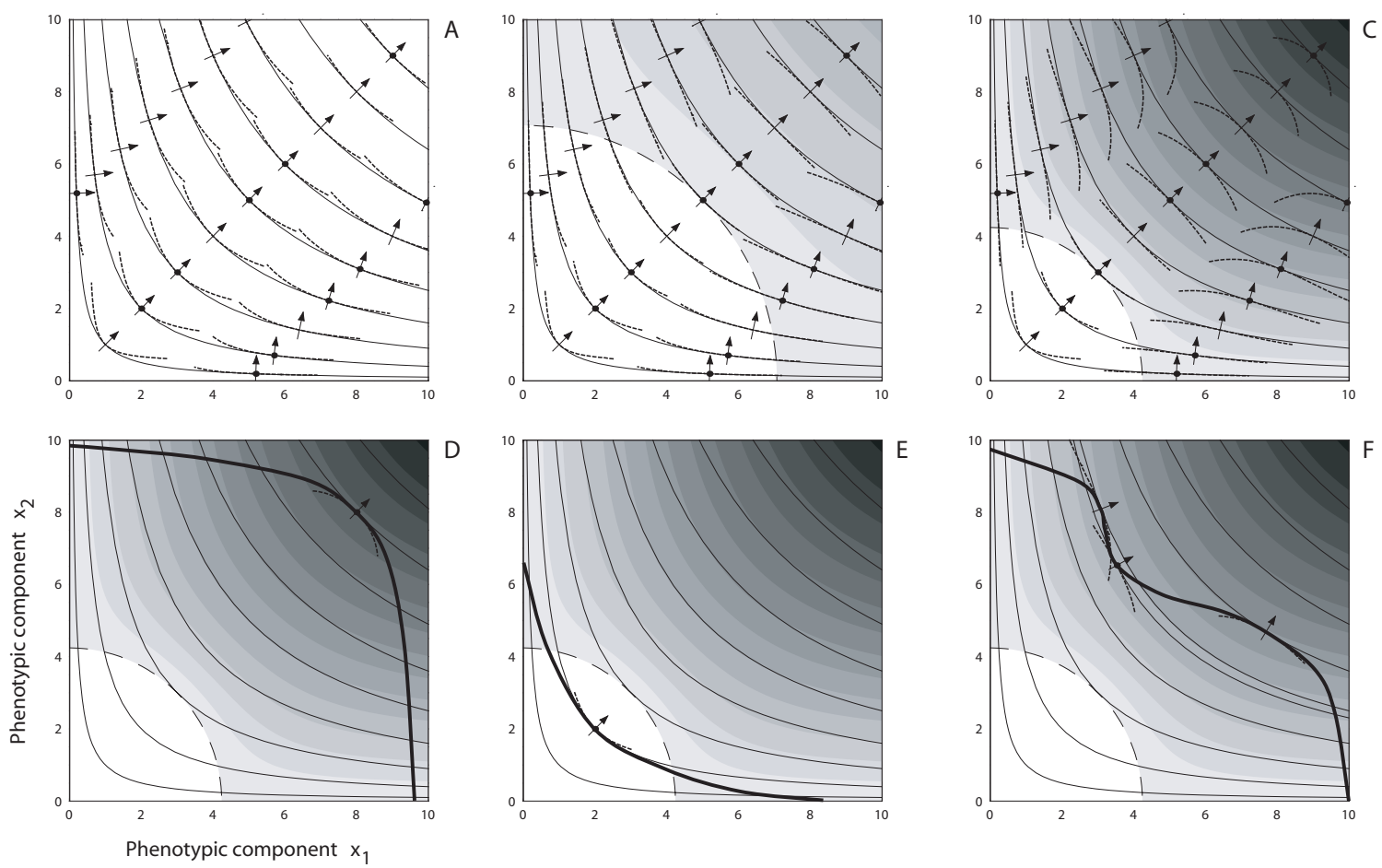

Figure 4 Geometric analysis of second example. The two phenotypic components $x_{1}$ and $x_{2}$ simultaneously affect carrying capacity and the strength of intraspecific competition. The degree of niche partitioning in both habitats is measured by $1 / \sigma^{2}$. Lines, arrows, and shading are as specified in figure 3 . (A) The case $\sigma^{2}=\infty$ corresponds to the absence of niche partitioning: evolutionary branching is impossible. For $\sigma^{2}<\infty$, all four types of evolutionarily singular strategies displayed in figure 3 can be induced by trade-off curves of suitable position and shape. Panels (B) with $\sigma^{2}=5$ and (C) with $\sigma^{2}=3$ show how the degree of niche partitioning affects the geometry of A-and I-boundaries, and thus the evolutionary outcomes that trade-offs can induce. Notice that no trade-off curve can induce evolutionary branching in the white area, which shrinks as niche partitioning increases; by contrast, only inside that area, trade-offs can induce Garden-of-Eden configurations. Panels (D) to (F) show evolutionary outcomes resulting from the selection pressures in panel (C) in conjunction with different trade-off curves. Other parameters used are $c_{1}=0.5$ and $k=r=1$. Further explanations concerning the second example are provided in Section 6.

phenotypes are highest for similar phenotypes $a_{i}\left(x^{\prime}, x\right)=\exp \left(-\frac{1}{2}\left(x_{i}^{\prime}-x_{i}\right)^{2} / \sigma^{2}\right)$, where $1 / \sigma^{2}$ measures the degree of niche partitioning in both habitats. With the equilibrium densities of a monomorphic resident population with phenotype $x$ thus given by $K_{1}(x)$ and $K_{2}(x)$, the invasion fitness of a rare phenotype $x^{\prime}$ is obtained as $f\left(x^{\prime}, x\right)=$ $c_{1} r\left[1-a_{1}\left(x^{\prime}, x\right) K_{1}(x) / K_{1}\left(x^{\prime}\right)\right]+c_{2} r\left[1-a_{2}\left(x^{\prime}, x\right) K_{2}(x) / K_{2}\left(x^{\prime}\right)\right]$. Contrary to the first example, the relative contribution of each habitat is thus no longer fixed, but instead varies with phenotype.

For $\sigma^{2}=\infty$, competitive outcomes between phenotypes only depend on the ratio of their carrying capacities. Once established, phenotypes with higher carrying capacities are better able to suppress the growth of potential invaders. Therefore, phenotypes that can be reached through gradual evolution cannot be reached through direct invasion. As shown in figure 4A, this leads to the possibility of Garden-of-Eden configurations. Niche partitioning occurs for $\sigma^{2}<\infty$ and yields an advantage to rarity, since competition between different phenotypes is then weaker than competition among them. Accordingly, the potential for encountering evolutionary branching points increases as 
$\sigma^{2}$ decreases; this is illustrated in figures 4B $(\sigma=5)$ and 4C $(\sigma=3)$. Figure 4D shows that, contrary to Levins's conclusion for evolution under frequency-independent selection, polymorphisms can originate from a convex trade-off. As mentioned before, this only requires that the I-boundaries are also convex with a curvature lower than that of the trade-off curve. Figure 4E demonstrates that, again contrary to Levins's conclusion, the combination of a concave trade-off and concave fitness contours can result in monomorphic evolutionary outcomes. For this to happen, the concave A- and Iboundaries just have to possess a larger curvature than the trade-off curve. Finally, figure $4 \mathrm{~F}$ illustrates how trade-offs with more intricate shapes can induce complex forms of evolutionary bistability, in this case between a continuously stable strategy and an evolutionary branching point.

All conclusions obtained above through geometric inspection can be derived analytically, using the tools described in Appendix 1.

\section{Discussion}

We have introduced a systematic framework for geometrically understanding constrained two-dimensional life-history evolution under frequency-dependent selection. The method enables to visualize in phenotypic space three important characteristics of frequency dependent selection: A-boundaries show phenotypes attainable by small evolutionary steps, I-boundaries show phenotypes that can invade an established resident, and shading shows the potential for evolutionary branching (as in figures $3 \mathrm{~A}-3 \mathrm{~B}$ and figures $4 \mathrm{~A}-4 \mathrm{C}$ ). Possible trade-off curves can then be superimposed to visualize evolutionary outcomes easily (as in figures $3 \mathrm{C}-3 \mathrm{D}$ and figures $4 \mathrm{D}-4 \mathrm{~F}$ ). As the particular shapes of most life-history trade-offs are uncertain and often controversial, we propose the following advantages of this analysis: (i) evolutionary implications of all relevant trade-off shapes can be analyzed in one go, (ii) evolutionary outcomes overlooked in earlier analysis based on specific trade-offs can be revealed, (iii) evolutionary conclusions are robust to variations within the classes of trade-off shapes delineated by the analysis itself, and (iv) qualitative effects of environmental changes on evolutionary outcomes can be derived independently of the trade-off considered. Our framework thus brings a vast range of fundamental life-history questions involving frequency dependence into the remit of a geometric method that can separate between the evolutionary implications of selection pressures and of adaptive constraints. Any life-history trade-off involving two evolutionarily variable continuous phenotypes can thus be investigated. Naturally, life-history trade-offs critically involving three or more independent phenotypic components are not amenable to a geometric analysis based on two-dimensional diagrams.

It may sometimes be desirable to extend the approach presented here to situations in which the gradual evolution of a two-dimensional phenotype $x$ is described by $d x / d t \infty v(x) \cdot g(x)$, where $g$ is the local selection gradient and $v$ equals either a population's genetic variance-covariance matrix (quantitative genetics approach) or the variance-covariance matrix assumed for the distribution of evolutionary innovations (adaptive dynamics approach). Our geometric framework can accommodate this generalization simply by plotting the vectors $v \cdot g$ instead of $g$ in diagrams like figures 
3 and 4. Notice that, while this may affect which parts of a trade-off curve or which interior singular phenotypes are evolutionarily attracting (Leimar 2001), evolution along a trade-off curve is one-dimensional and consequently does not involve a variancecovariance matrix.

Evolutionary responses to frequency-dependent disruptive selection, and thus establishment of population-level polymorphisms, differ in models based, alternatively, on quantitative genetics or adaptive dynamics. In general, asexual populations are bound to become bimodal in response to disruptive selection, while such evolution may be prevented in sexual populations through segregation and recombination (Felsenstein 1981). In the quantitative genetics approach based on Lande (1979), the assumption of normally distributed phenotypes with constant variances precludes evolution of bimodality; this approach is thus more geared to sexual organisms. By contrast, the bimodalities that can arise in asexual organisms are well described by the adaptive dynamics approach. Yet, it has recently been highlighted that sexual populations can also become bimodal through frequency-dependent disruptive selection as long as mating between the two modes is (or evolves to become) sufficiently assortative (e.g., Johnson et al. 1996; Dieckmann and Doebeli 1999). Addressing questions of this nature requires genetically explicit modeling, which is beyond our scope here.

Finally, we would like to draw attention to the power of the analytical counterpart of the geometric approach introduced here. Especially for life-history models involving a multitude of parameters or trade-offs, it can be advantageous to replace the visual inspection of diagrams by derivations and proofs. Appendix 1 provides the tools necessary for such analyses. 


\section{Box 1 Glossary of key terms}

Frequency-dependent selection (or frequency dependence, for short) causes the fitness of a phenotype to depend on the phenotypes and phenotypic frequencies of conspecifics.

Adaptive dynamics theory allows analyzing the course of phenotypic evolution resulting from the invasion and fixation of mutational innovations under conditions of frequency-dependent selection. A simplifying assumption often made in such analyses is that evolution is asexual and mutation-limited. This results in populations being monomorphic in between invasion and fixation events, composed of a resident phenotype at ecological equilibrium. Although the resident is assumed to reach its demographic equilibrium before a new mutant comes along, simulations shows that the predictions are fairly robust to a relaxation of this assumption (Dieckmann et al. 1995; Geritz et al. 1998). When invasion occurs, the new phenotype replaces the previous resident. Density-dependent selection affects adaptive dynamics when ecological equilibria depend on resident phenotypes, and is thus naturally accounted for in adaptive dynamics theory.

Quantitative genetics theory allows analyzing the course of evolution in polymorphic distributions of quantitative trait values. Simplifying assumptions frequently made in such analyses are that quantitative traits are normally distributed and that the corresponding variances remain constant over evolutionary time. In this way attention can be restricted to the evolution of mean phenotypes.

Invasion fitness of a phenotype is defined as its growth rate while being rare in a population of given phenotypic composition. It is key to any analysis of frequencydependent selection.

Local selection gradients point in the phenotypic direction in which invasion fitness increases most steeply around the phenotypic mode of a resident population.

An evolutionarily singular strategy is a phenotype for which (realizable) local selection gradients vanish. This means that, locally, the selection gradient points towards the trade-off curve and the gradient's component orthogonal to that curve vanishes. A singular strategy is either convergence stable or unstable and is either evolutionarily stable or unstable.

A convergence stable strategy is a strategy towards which directional evolution will converge through small evolutionary steps.

An evolutionarily stable strategy (ESS) is a strategy that, once resident, cannot be invaded by any other strategy.

A continuously stable strategy is a singular strategy that is both locally evolutionarily stable and convergence stable, and thus acts as a likely endpoint of directional evolution.

An invasible repellor is a singular strategy that lacks both local evolutionary stability and convergence stability. Evolution leads away from such a repellor, resulting in evolutionary bistability.

A Garden-of-Eden configuration is a singular strategy that is locally evolutionarily stable (and hence, once attained, uninvasible by neighboring strategies) while at the same time not convergence stable (and hence not attainable through small evolutionary 
steps). This implies evolutionary multistability, which in this case also includes, as a third possible outcome, the singular strategy itself.

An evolutionary branching point is a singular strategy that is convergence stable but lacks local evolutionary stability. It causes evolutionary convergence to disruptive selection, which can result in a population-level protected dimorphism.

An attainability boundary (A-boundary) separates areas of trait space that can and cannot be invaded starting from a focal phenotype through successive invasion and replacement of resident strategies by neighboring mutant strategies.

An invasibility boundary (I-boundary) separates areas of trait space that can and cannot invade into a population composed of a focal phenotype. Contrary to an Aboundary, which applies to all points through which it passes, an I-boundary only applies to its focal phenotype.

A trade-off curve separates areas of trait space that contain and do not contain feasible phenotypes. 


\section{Appendices}

\section{Appendix 1: Analytical methods}

Analysis of constrained life-history evolution of phenotypes $x=\left(x_{1}, x_{2}\right)$ under frequency-dependent selection is based on a function $f\left(x^{\prime}, x\right)$ describing invasion fitness, and on one or more trade-off curves. The local selection gradient resulting from $f$ is $g(x)=\left(g_{1}(x), g_{2}(x)\right)=\left.\left(\partial f / \partial x_{1}^{\prime}, \partial f / \partial x_{2}^{\prime}\right)\right|_{x^{\prime}=x}$.

A trade-off curve $\mathrm{T}=\left\{\left(x_{T 1}, x_{T 2}\right)\right\}$ demarcates the feasible part of trait space. It can either be represented explicitly, $x_{T 2}=T\left(x_{T 1}\right)$, or parametrically, $x_{T}=\left(\tau_{1}(p), \tau_{2}(p)\right)$. Its slope at $x_{T}$ is $S_{T}=T^{\prime}\left(x_{T 1}\right)$ or $S_{T}=\tau_{2}^{\prime}(p) / \tau_{1}^{\prime}(p)$, and its curvature there is $C_{T}=\omega T^{\prime \prime}\left(x_{T 1}\right)\left[1+T^{\prime 2}\left(x_{T 1}\right)\right]^{-3 / 2}$ or

$C_{T}=\omega\left[\tau_{1}^{\prime}(p) \tau_{2}^{\prime \prime}(p)-\tau_{1}^{\prime \prime}(p) \tau_{2}^{\prime}(p)\right]\left[\tau_{1}^{\prime 2}(p)+\tau_{2}^{\prime 2}(p)\right]^{-3 / 2}$ (Lelong-Ferrand and Arnaudies 1977). The orientation factor $\omega$ realizes our sign convention for curvatures, $\omega=\operatorname{sgn} g_{2}(x)$.

The A-boundary of phenotype $x$, denoted $A(x)=\left\{\left(x_{A 1}, x_{A 2}\right)\right\}$, demarcates the region of phenotypes reachable from $x$ through gradual directional evolution. Being everywhere orthogonal to the local selection gradient $g$, it has the slope of $g$ 's normal and hence solves the differential equation $x_{A 2}^{\prime}\left(x_{A 1}\right)=-g_{1}\left(x_{A}\right) / g_{2}\left(x_{A}\right)$ with initial condition $x_{A 2}\left(x_{1}\right)=x_{2}$. Accordingly, its slope at $x$ is $S_{A}=-g_{1}(x) / g_{2}(x)$ and its curvature there is $C_{A}=\omega\left(\partial S_{A} / \partial x_{1}+S_{A} \partial S_{A} / \partial x_{2}\right)\left(1+S_{A}^{2}\right)^{-3 / 2}$.

The I-boundary of $x, I(x)=\left\{x_{I} \mid f\left(x_{I}, x\right)=0\right\}$, demarcates the invader set of $x$, i.e., the region of phenotypes able to invade $x$. Its slope at $x$ is $S_{I}=S_{A}$, and its curvature there is

$C_{I}=-\left.\omega\left(\partial^{2} f / \partial x_{1}^{\prime 2}+2 S_{I} \partial^{2} f / \partial x_{1}^{\prime} \partial x_{2}^{\prime}+S_{I}^{2} \partial^{2} f / \partial x_{2}^{\prime 2}\right)\left(\partial f / \partial x_{2}^{\prime}\right)^{-1}\left(1+S_{I}^{2}\right)^{-3 / 2}\right|_{x^{\prime}=x}$.

A phenotype $x_{T}$ along a trade-off curve is evolutionarily singular iff $S_{A}=S_{I}=S_{T}$ at $x_{T}$. It is convergence stable iff $C_{T}<C_{A}$ at $x_{T}$, and it is locally evolutionarily stable iff $C_{T}<C_{I}$ at $x_{T}$. This means that $x_{T}$ is a continuously stable strategy iff $C_{T}<C_{A}, C_{I}$, an invasible repellor iff $C_{A}, C_{I}<C_{T}$, a Garden-of-Eden configuration iff $C_{A}<C_{T}<C_{I}$, and an evolutionary branching point iff $C_{I}<C_{T}<C_{A}$. In particular, evolutionary branching points can only occur at phenotypes $x_{T}$ at which $C_{A}-C_{I}>0$.

Some models may incorporate more than a single trade-off, which may cause evolution to converge on interior attractors without ever being constrained by the focal trade-off. Analyses of multi-dimensional adaptive dynamics are then required; these are not the focus of the present paper and are addressed elsewhere (Dieckmann and Law 1996; Meszena et al. 2001; Leimar 2001).

\section{Appendix 2: Classical fitness set analysis}

Richard Levins's fitness set analysis was inspired by his interest in the adaptation of organisms exposed to heterogeneous environments. Levins initially focused on the situation in which an organism has access to two habitats or niches requiring differential adaptation. By considering an organism's performance in these two environments, Levins defined a two-dimensional trait space, spanned by the components of fitness in each environment. Two different quantities are then considered (Levins 1962a): 

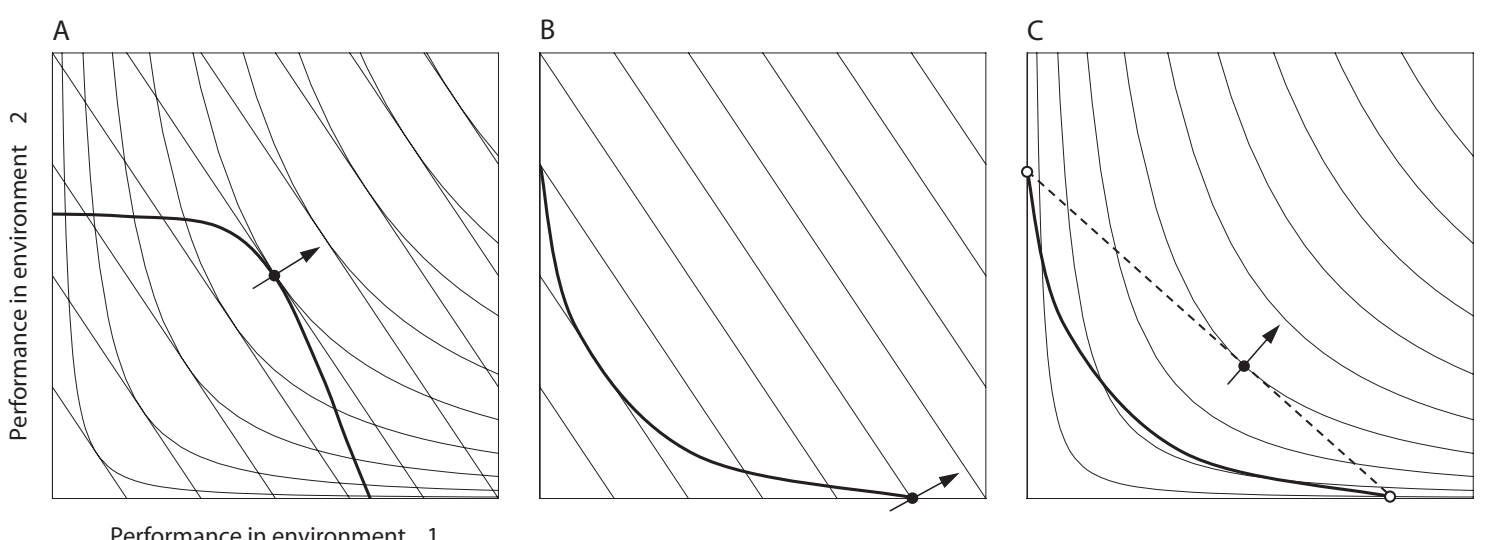

Figure 5 Evolutionary outcomes in Levins's original geometric analysis. Thin continuous lines are contours of the adaptive function, while thick lines are fitness sets. Arrows indicate the direction of the local selection gradient, and filled circles show the evolutionary outcomes. (A) If the fitness set is convex, a single generalist evolves. (B) If the fitness set is concave and the adaptive function linear, a single specialist evolves. (C) If the fitness set is concave and the adaptive function hyperbolic, a mixture of two specialist strategies evolves.

- First is the fitness set, which simply is the subset of trait space allowed by a tradeoff or constraint. Only combinations of performances lying inside the fitness set are feasible and can thus be reached by evolution of those performances.

- Second is the adaptive function. Restricting attention to frequency-independent selection, this function is defined for all points in trait space and measures the organism's fitness in the heterogeneous environment resulting from its performance in the two separate habitats.

Relying on the notion of adaptive evolution maximizing frequency-independent fitness, Levins reached the following conclusions about expected evolutionary outcomes:

- When the fitness set is convex (looking along the main diagonal towards the origin), a single generalist evolves (figure 5A).

- When the fitness set is concave and the adaptive function is linear, a single specialist evolves (figure 5B).

- When the fitness set is concave and the adaptive function is hyperbolic, a mixture of two specialist strategies evolves (figure 5C). This can either correspond to an individual-level mixed strategy (in which each individual probabilistically switches between two pure strategies) or to a population-level mixed strategy (in which two different phenotypes coexist in a dimorphism). Like in evolutionary game theory (Maynard Smith 1982), these two biologically quite distinct situations are formally equivalent in Levins's approach.

\section{Appendix 3: Comparison of approaches}

Two critical modifications are required for extending Levins's geometric approach to frequency-dependent selection. First, components of fitness can no longer be used as axes defining a population's trait space: since the fitness of an organism no longer only depends on its own phenotype but also on those in the remainder of its population, fitness components no longer uniquely characterize a phenotype. Our new framework is therefore directly based on the underlying phenotypic traits; these same axes are also 
traditionally used to define adaptive landscapes. As a result of this change of axes, Levins's fitness sets are replaced by the more general notion of trade-off curves. Second, to describe frequency-dependent selection we have to replace Levins's adaptive function by the more general notion of invasion fitness. Since fitness contours dynamically vary as evolution proceeds, static contours - a key feature in Levins's geometric analysis - are unavailable under frequency-dependent selection. Only when frequency-dependent selection is absent, the I- and A-boundaries introduced in the present paper coincide and concur with the contours of Levins's static adaptive function.

Compared to Levins's original classification of evolutionary outcomes (Appendix 2 ), we must draw attention to two important changes relative to his conclusions. First, even when trade-off curves are convex, evolution can become polymorphic - we only need $C_{I}<C_{T}<C_{A}$ (figure 4D). Second, even when trade-off curves and fitness contours are concave, evolution can remain monomorphic - we only need $C_{T}<C_{I}, C_{A}$ (figure 4E.). 


\section{References}

Abrams PA \& Matsuda H (1996). Fitness minimization and dynamic instability as a consequence of predator-prey coevolution. Evolutionary Ecology 10:167-186

Abrams PA, Matsuda H \& Harada Y (1993). Evolutionarily unstable fitness maxima and stable fitness minima of continuous traits. Evolutionary Ecology 7: 465-487

Calow P (1999). Encyclopedia of Ecology and Environmental Management. Blackwell Publishing

Case TJ (1999). An Illustrated Guide to Theoretical Ecology. Oxford, UK: Oxford University Press

de Mazancourt C, Loreau M \& Dieckmann U (2001). Can the evolution of plant defense lead to plant-herbivore mutualism? The American Naturalist 158: 109-123

de Meeus T. \& Goudet J. (2000). Adaptive diversity in heterogeneous environments for populations regulated by a mixture of soft and hard selection. Evolutionary Ecology Research 2: 981-995

Dieckmann U \& Doebeli M (1999). On the origin of species by sympatric speciation. Nature 400: 354-357

Dieckmann U \& Law R (1996). The dynamical theory of coevolution: A derivation from stochastic ecological processes. Journal of Mathematical Biology 34: 579612

Dieckmann U (1997). Can adaptive dynamics invade? Trends in Ecology and Evolution 12: $128-131$

Dieckmann U (2002). Adaptive dynamics of pathogen-host interactions. In: Dieckmann U, Metz JAJ, Sabelis MW, Sigmund K (eds) Adaptive Dynamics of Infectious Diseases: In Pursuit of Virulence Management, pp. 39-59. Cambridge, UK: Cambridge University Press

Dieckmann U, Marrow P \& Law R (1995). Evolutionary cycling in predator-prey interactions: Population dynamics and the Red Queen. Journal of Theoretical Biology 176: 91-102

Ebert D \& Bull JJ (2003). Challenging the trade-off model for the evolution of virulence: Is virulence management feasible? Trends in Microbiology 11: 15-20

Felsenstein J (1981). Skepticism towards Santa Rosalia, or why are there so few kinds of animals? Evolution 35: 124-138

Ferrière R \& Gatto M (1995). Lyapunov exponents and the mathematics of invasion in oscillatory or chaotic populations. Theoretical Population Biology 48: 126-171

Geritz SAH, Kisdi É, Meszena G \& Metz JAJ (1998). Evolutionarily singular strategies and the adaptive growth and branching of the evolutionary tree. Evolutionary Ecology 12: 35-57 
Geritz SAH, Metz JAJ, Kisdi É \& Meszéna G (1997). Dynamics of adaptation and evolutionary branching. Physical Review Letters 78: 2024-2027

Harding SP \& Lovelock JE (1996). Exploiter-mediated coexistence and frequencydependent selection in a numerical model of biodiversity. Journal of Theoretical Biology 182: 109-116

Hauert C, De Monte S, Hofbauer J \& Sigmund K (2002). Volunteering as Red Queen mechanism for co-operation in public goods games. Science 296: 1129-1132

Iwasa Y, Pomiankowski A \& Nee S (1991). The evolution of costly mate preferences. II. The "handicap" principle. Evolution 45: 1431-1442

Johnson PA, Hoppensteadt FC, Smith JJ \& Bush GL (1996). Conditions for sympatric speciation: A diploid model incorporating habitat fidelity and non-habitat assortative mating. Evolutionary Ecology 10: 187-205

Kisdi É \& Geritz SAH (2001). Evolutionary disarmament in interspecific competition. Proceedings of the Royal Society of London B 268: 2589-2594

Kisdi É (1999). Evolutionary branching under asymmetric competition. Journal of Theoretical Biology 197: 149-162

Kisdi É (2001). Long-term adaptive diversity in Levene-type models. Evolutionary Ecology Research, 3: 721-727

Lande R (1976). Natural selection and random genetic drift in phenotypic evolution. Evolution 30:314-334.

Lande R (1979). Quantitative genetic analysis of multivariate evolution, applied to brain:body size allometry. Evolution 33: 402-416

Law R (1979). Optimal life histories under age-specific predation. The American Naturalist 114: 399-417

Law R, Marrow P \& Dieckmann U (1997). On evolution under asymmetric competition. Evolutionary Ecology 11: 485-501

Le Galliard JF, Ferrière R \& Dieckmann U (2003). The adaptive dynamics of altruism in spatially heterogeneous populations. Evolution 57: 1-17

Leimar O (2001). Evolutionary change and Darwinian demons. Selection 2: 65-72

Lelong-Ferrand J \& Arnaudies JM (1977). Cours de mathématiques. Tome 3. Géométrie et cinématique. Dunod, Paris

Levins R (1962a). Theory of fitness in a heterogeneous environment. I. The fitness set and adaptive function. The American Naturalist 96: 361-373

Levins R (1962b). Theory of fitness in a heterogeneous environment. II. Developmental flexibility and niche selection. The American Naturalist 97: 74-90

Levins R (1968). Evolution in changing environments. Some theoretical explorations. Princeton, USA: Princeton University Press 
Lipsitch M, Siller S \& Nowak MA (1996). The evolution of virulence in pathogens with vertical and horizontal transmission. Evolution 50: 1729-1741

Mallet J (1999). Causes and consequences of a lack of coevolution in Mullerian mimicry. Evolutionary Ecology 13: 777-806

Matsuda H \& Abrams PA (1994). Runaway evolution to self-extinction under asymmetrical competition. Evolution 48: 1764-1772

May RM (1983). Parasitic infections as regulators of animal populations. American Scientist 71: 36-45

Maynard Smith J (1982). Evolution and the Theory of Games. Cambridge, UK: Cambridge University Press

Meszéna G, Kisdi É, Dieckmann U, Geritz SAH \& Metz JAJ (2001). Evolutionary optimisation models and matrix games in the unified perspective of adaptive dynamics. Selection 2: 193-210

Metz JAJ, Geritz SAH, Meszéna G, Jacobs FJA \& van Heerwaarden JS (1996). Adaptive dynamics: A geometrical study of the consequences of nearly faithful reproduction. In: van Strien SJ \& Verduyn Lunel SM (eds) Stochastic and Spatial Structures of Dynamical Systems, pp. 183-231. Amsterdam, Netherlands: NorthHolland

Metz JAJ, Nisbet RM \& Geritz SAH (1992). How should we define "fitness" for general ecological scenarios? Trends in Ecology and Evolution 7: 198-202

Mole S (1994). Trade-offs and constraints in plant-herbivore defense theory: A lifehistory perspective. Oikos 71: 3-12

Nowak MA, Bonhoeffer S \& May RM (1994). Spatial games and the maintenance of cooperation. Proceedings of the National Academy of Science USA 91: 48774881

Rand DA, Wilson HB \& McGlade JM (1993). Dynamics and evolution: Evolutionarily stable attractors, invasion exponents and phenotype dynamics. Philosophical Transactions of the Royal Society of London B 343: 261-283

Rueffler C, Van Dooren TJM \& Metz JAJ (2004). Adaptive walks on changing landscapes: Levins' approach extended. Theoretical Population Biology, 65: 165178.

Saloniemi I (1993). A coevolutionary predator-prey model with quantitative characters. The American Naturalist 141: 880-896

Sigmund K (2002). The economics of fairness. Scientific American 286: 82-87

Simms EL \& Rausher MD (1987). Costs and benefits of plant resistance to herbivory. The American Naturalist 130: 570-581

Taper ML \& Case TJ (1985). Quantitative genetic models for the coevolution of character displacement. Ecology 66: 355-371 
Taper ML \& Case TJ (1992). Models of character displacement and the theoretical robustness of taxon cycles. Evolution 46: 317-333

Turelli M \& Barton NH (1994). Genetic and statistical analyses of strong selection on polygenic traits - What, me normal? Genetics 138: 913-941

Vincent TL, Cohen Y \& Brown JS (1993). Evolution via strategy dynamics. Theoretical Population Biology 44: 149-176

Yodzis P (1989). Introduction to Theoretical Ecology. New York: Harper \& Row 